\title{
¿HAY ALGUIEN EN LÍNEA? : VALIDEZ Y FIABILIDAD DE LA VERSIÓN EN ESPAÑOL DE LA BERGEN FACEBOOK ADDICTION SCALE (BFAS) EN UNIVERSITARIOS
}

\section{IS THERE ANYONE ONLINE? : VALIDITY AND RELIABILITY OF THE SPANISH VERSION OF THE BERGEN FACEBOOK ADDICTION SCALE (BFAS) IN UNIVERSITY STUDENTS}

\author{
Miguel Ángel Vallejos-Flores ${ }^{1,3}$, Anthony Copez-Lonzoy ${ }^{2,3}$ y Walter Capa-Luque ${ }^{1}$ \\ 'Universidad Nacional Federico Villarreal, Lima, Perú \\ 2 Universidad de San Martín de Porres, Lima, Perú \\ ${ }^{3}$ Asociación Peruana de Profesionales de las Adicciones, Lima, Perú
}

\section{Abstract}

The impact of social networks on people's daily lives is alarming, especially among adolescents and young people, which seems to exceed the limits of normal use and to be oriented towards pathological patterns that could lead to an addictive disorder. The objective of this study was to provide evidence of validity and reliability of BFAS scores. A sample of 510 university students was used and the average age of the participants was 20.74 years $\left(S D_{\text {age }}=2.57\right)$ and $54.1 \%$ were women. For the Bergen Facebook Addiction Scale (BFAS) the questionnaire Fear of Missing Out (FoMO) was applied as a measure of contrast $(r=.60)$. The internal structure was determined under the confirmatory factorial analysis (CFA), the equivalence of measurement was also evaluated by multigroup factor analysis models (CFA-MG) where levels of invariance were verified by sex, age and academic involvement. The relevant use of the alpha coefficient was corroborated by the implementation of restrictions in confirmatory models to evaluate tauequivalence and parallel measures, where adequate results were obtained $(a=.84)$. This version of the BFAS reports useful evidence for research into behavioral addictions due to its need to be included in batteries that assess addictions to social networks.

Keywords: addiction, measurement equivalence, Facebook, reliability, validity

\section{Resumen}

El impacto de las redes sociales en la vida cotidiana de las personas es preocupante, sobre todo en los adolescentes y jóvenes, que parece rebasar los límites del uso normal y orientándose hacia patrones patológicos que podrían conllevar a un trastorno adictivo. El objetivo del presente estudio fue brindar evidencias de validez y fiabilidad de las puntuaciones de la escala BFAS. Se utilizó una muestra de 510 jóvenes universitarios y la edad promedio de los participantes fue de 20.74 años ( $D E_{\text {edad }}=2.57$ ) y el $54,1 \%$ fueron mujeres. Para la Bergen Facebook Addiction Scale (BFAS) se aplicó el cuestionario Fear of Missing Out (FoMO) como medida de contraste $(r=.60)$. La estructura interna fue determinada bajo el análisis factorial confirmatorio (AFC), también fue evaluada la equivalencia de medición mediante modelos de análisis factorial multigrupo (AFCMG) donde se comprobaron niveles de invarianza por sexo, edad y afectación académica. Se corroboró el uso pertinente del coeficiente alfa mediante la implementación de restricciones en modelos confirmatorios para evaluar tau-equivalencia y medidas paralelas, donde se obtuvo resultados adecuados ( $\alpha=.84)$. Esta versión del BFAS reporta evidencias útiles para la investigación en adicciones comportamentales debido a su necesidad de inclusión en baterías que evalúen adicciones a redes sociales.

Palabras clave: adicción, equivalencia de medición, Facebook, fiabilidad, validez. 
El impacto de las redes sociales virtuales (RSV) en la vida cotidiana ha ido en un aumento progresivo por lo que se ha convertido en una situación controversial establecer límites entre el uso normal y patológico. Es innegable que las RSV son herramientas indispensables para el desarrollo humano y social generando un sinfín de beneficios, pero también el uso inadecuado de estas plataformas está generando algunos problemas psicopatológicos de carácter novedoso como las ciberdislexias, ciberbullying, nomofobia, miedo a estar desinformado y ciberadicciones, que están agudizando otros problemas relacionados como la autoestima, impulsividad, narcisismo, entre otros (Dias, Cadime, del Castillo-López, García-Castillo, y García del Castillo, 2018; El Sahili, 2015; Garcia del Castillo, 2013).

Un hecho distintivo de la última década es el gran avance de las RSV y sus repercusiones en la sociedad. No cabe duda que la RSV de mayor desarrollo e impacto es Facebook con más de dos billones de usuarios activos por mes (Dias et al., 2018; El Sahili, 2015). Este uso cotidiano y frecuente puede conllevar a la presencia de un trastorno adictivo en muchos usuarios, tarea frecuente a la que se enfrenta un especialista de la salud mental en su práctica profesional y que a la vez se ha convertido en una de las prioridades de las estrategias de salud a nivel internacional, problema que está generando gran costo sociosanitario.

Dentro de esta línea, las ciberadicciones han tomado relevancia en la investigación, debido a su relación, predicción y riesgo asociado con otras variables tecnológicas como es el uso de redes sociales (Alt, 2015; Przybylski, Murayama, DeHaan y Gladwell, 2013; Roberts y David, 2016; Yildirim y Correia, 2015). El comportamiento adictivo se caracteriza por la pérdida de control generando en la persona consecuencias en su entorno familiar, académico y social (Becoña y Cortés, 2011). En este contexto, los adolescentes y jóvenes representan una población vulnerable debido a que usan esta plataforma como una herramienta interactiva para el desarrollo de diversas actividades (como es el caso de escolares y universitarios), sin embargo la continua actualización de noticias, el incremento de usuarios, las conversaciones espontáneas con sus pares y diversos estímulos distractores son variables que mantienen el uso frecuente a esta RSV que contribuye al proceso adictivo y por ende consecuencias negativas en el repertorio conductual caracterizado por la relevancia, tolerancia, cambios en el estado de ánimo, síntomas de abstinencia, presencia de conflictos ocasionado por el uso intensivo y recaídas (Andreassen, 2015; Hormes, Kears y Timko, 2014).

Este comportamiento adictivo es de mayor preocupación por las consecuencias negativas que acarrea en la salud del usuario (depresión, ansiedad, estrés, trastorno del sueño, entre otros) como sugieren algunos estudios transversales (Brailovskaia y Margraf, 2017). Por ello este problema ha generado preocupación en los investigadores debido a las consecuencias legales relacionados a la adicción del Facebook (Drouin, O'Connor, Schmidt y Miller, 2015), como predictor de conductas procastinadoras en universitarios (Meier et al., 2016), bienestar y uso problemático del Facebook (Satici y Uysal, 2015), uso adictivo a Facebook como conducta escapista (Young, Kuss, Griffiths y Howard, 2017), condiciones clínicas asociadas con altas puntuaciones en adicción al Facebook implicadas a la necesidad de admiración y grandiosidad (Casale y Fioravanti, 2018), neuroticismo y psicoticismo (Wang, Ho, Chan y Tse, 2015), ansiedad social y síntomas depresivos asociados al uso problemático del Facebook (Moreau, Laconi, Delfour y Chabrol, 2015) e incluso consumo de alcohol y tabaco relacionados con uso abusivo del Facebook (Hormes et al., 2014) convirtiéndose estas condiciones en factores de mantenimiento para su uso perjudicial y adictivo (Dhaha, 2013; Meier, Reinecke y Meltzer, 2016).

Sumado a lo anterior, uno de los detonantes de la adicción al Facebook, es el miedo a estar desinformado o de perderse de algo (FoMO), debido a que genera conductas de irritabilidad, ansiedad y sentimientos desadaptativos que se refuerzan cada vez que el individuo se conecta constantemente a las redes sociales, ya que los usuarios suelen disfrutar estar enterados de alguna noticia presentada en estas redes con el fin de mantenerse "al día"; de manera contraria al no actualizarse de las nuevas publicaciones presentará efectos negativos que pueden influir en su estado de ánimo afectando su autoeficacia , autonomía y relación con los demás, así como el aumento de sentimientos de indignación y ansiedad por la idea de perderse alguna noticia (Abel, Buff, y Burr, 2016). El FoMO es un factor potenciador que impulsa la conducta de conexión (uso y abuso) al aumentar la participación en las redes sociales, especialmente en el grupo de los jóvenes que constantemente actualizan sus RSV y retroalimentan a sus pares siguiendo un ciclo de conexión (Abel et al., 2016; Alt, 2015). 
Esta realidad demanda a los profesionales de contar con herramientas diagnósticas y terapéuticas para el abordaje temprano de las adicciones tecnológicas que conlleven a la reducción de costos personales y sociofamiliares. Diversos estudios reportan que uno de los instrumentos más usado para la medición de la adicción al Facebook es la Bergen Facebook Addiction Scale - BFAS o bien su versión traducida al tailandés y recientemente al portugués; estos estudios indicarían en cierta medida la adicción a Facebook es uno de los fenómenos más estudiados en países europeos y asiáticos, con escasos estudios en países hispanohablantes (Dias et al., 2018; El Sahili, 2015; Satici y Uysal, 2015). Es así que la BFAS se ha convertido en el instrumento de mayor uso para estudiar la adicción a Facebook y que ha evidenciado mejores índices de validez y fiabilidad de sus puntuaciones (Andreassen, 2015; Andreassen, Torsheim, Brunborg y Pallesen ,2012; Dias et al., 2018; Pontes, 2016; Salem, Almeye y Andreassen, 2015; Satici y Uysal 2015; Silva et al., 2015). Originalmente este instrumento fue construido con base de 18 ítems y constituido enteramente por una muestra de universitarios noruegos $(n=423)$ donde fueron reportadas relaciones moderadas y altas con la escala de tendencias adictivas al Facebook ( $r=69)$ y la escala de actitudes al Facebook ( $r=.58$ ). A nivel estructural fueron puestos a prueba diferentes modelos confirmatorios, finalmente la solución fue representada por seis ítems (CFI=.99; RMSEA=.05). El coeficiente de correlación testretest después de 3 semanas $(n=153)$ fue de .82 con intervalos de confianza al 95\% $[0,75,0,86](p<.01)$ y la fiabilidad de las puntuaciones por consistencia interna fue óptima ( $a=83$ ). Estudios en Asia, Europa y en Brasil (Ahmet, Demirci, y Serap, 2017; Andreassen et al., 2012; Silva et al., 2015) han evaluado sus propiedades psicométricas, donde prevalece la unidimensionalidad de la escala, además la fiabilidad supera el a>.80 que indicaría óptimos resultados a nivel de precisión de las puntuaciones en población universitaria (Ahmet et al., 2017; Andreassen, 2015; Andreassen et al., 2012; Dias et al., 2018; Phanasathit, Manwong, Hanprathet, Khumsri y Yingyeun, 2015; Salem et al., 2015; Satici y Uysal, 2015; Silva et al., 2015).

Ante la ausencia de instrumentos que evalúen este comportamiento adictivo surge la necesidad de analizar las propiedades psicométricas del BFAS para la detección temprana del riesgo de adicción al Facebook; sin embargo una de las dificultades hasta el momento es la ausencia de estudios instrumentales de una versión en español para la evaluación de la adicción al Facebook, insumo de utilidad para la identificación de conductas disruptivas, problemáticas y psicopatológicas relacionadas a esta adicción (Casale y Fioraventi, 2018; Dhaha, 2013; Drouin et al., 2015; Hormes et al., 2014; Meier et al., 2016; Satici y Uysal, 2015; Wang et al., 2015; Young et al., 2017). Es importante tomar en cuenta el reporte de las diferencias que pueden manifestarse en el comportamiento adictivo a redes sociales (por ejemplo, grupos de hombres y mujeres), que suelen ser un contraste con las adicciones a sustancias psicoactivas, debido a los rasgos específicos de personalidad que predisponen al trastorno adictivo con un mayor deterioro y la manifestación de estas diferencias según género para el abuso de sustancias, violencia, juegos de azar o comportamientos antisociales (Casale y Fioravanti 2018; Wang et al., 2015). Asimismo en los estudios sobre adicción al Facebook, en este factor diferencial han reportado que los usuarios varones pueden sentirse más atraídos a funciones de entretenimiento y las mujeres a la construcción de relaciones (Chan, Cheung, Shi y Lee, 2015). El estudio de Andreassen (2012) fue el único que reporta un buen ajuste sobre la interpretación del constructo entre hombres y mujeres a nivel de invarianza de cargas factoriales, (invarianza factorial). Sin embargo estas diferencias no necesariamente suelen mantenerse a nivel cultural y/o en el caso de otras adiciones comportamentales, tanto es así que solo el estudio original reporta un nivel inicial de invarianza, pero no se pudo evidenciar el cambio límite entre estos grupos (Andreassen et al., 2012; Becker, McClellan y Reed, 2017; Beiler-May, Williamson, Clark y Carter, 2017; Chang et al., 2015). Por otra parte, hasta la actualidad no hay estudios sobre la evaluación de equivalencia de medición en otros grupos objetivos relevantes como es el caso de la edad, debido a las claras diferencias entre etapas y sobre la percepción de la afectación a nivel académico (Meier et al., 2016; Ortuño-Sierra, Aritio-Solana y Fonseca-Pedrero, 2018)

El objetivo del presente estudio fue analizar las evidencias de validez de la estructura interna, fiabilidad de las puntuaciones y equivalencia de medición de la escala BFAS en jóvenes universitarios. Este trabajo se justifica por ser la primera versión en español del instrumento, su pertinente uso en el estudio de las adicciones a redes sociales y su contribución en otros posibles estudios relacionados en esta línea de investigación. 


\section{MÉTODO}

\section{Participantes}

La muestra del estudio estuvo conformada por 510 jóvenes universitarios peruanos. El proceso de acceso a la muestra se realizó mediante el muestreo por conveniencia. El 54.1\% de la muestra fueron mujeres, con una media de edad de 20.74 ( $\left.D E_{\text {edad }}=2.57\right)$, asimismo el $45,9 \%$ de los participantes informaron que el uso prolongado del Facebook había afectado en sus estudios, al $22,9 \%$ en sus relaciones y al $28.1 \%$ en sus relaciones familiares. Todos los participantes refirieron que usan Facebook como principal red social.

\section{Instrumentos}

Bergen Facebook Addiction Scale (BFAS) es desarrollada por Andreassen (Andreassen et al., 2012), es una escala unidimensional que consta de seis ítems, con un escalamiento de cinco opciones de respuesta ( $1=$ muy raramente, $2=$ raramente, $3=$ a veces, $4=$ a menudo, 5= muy a menudo), la puntuación total consta de la sumatoria de todos los ítems para la adicción al Facebook. Cuenta con una replicación a nivel de su estructura interna con un solo factor y análisis por consistencia interna superior a .80 .

El proceso de adaptación lingüística para la escala BFAS fue realizado bajo la estrategia de traducción inversa siguiendo los controles de calidad recomendados por las directrices para la adaptación de instrumentos de medida (Muñiz, Elosua, y Hambleton, 2013). En primer lugar, fueron reclutados traductores con experiencia en procesos psicométricos para evaluar aspectos gramaticales y de redacción de los ítems que fueron considerados para el idioma español. La traducción del BFAS fue realizada del inglés al español considerando la versión original en inglés (Andreassen et al., 2012) y del español al inglés donde el juicio de la traducción fue evaluada cualitativamente por los expertos a partir de las traducciones realizadas para la equivalencia de las versiones propuesta por los traductores, donde fueron comparadas tres versiones, para desarrollar la versión final en español.

Fear of Missing Out (FoMO), desarrollada por Przybylski, Murayama, DeHann y Gladwell (Przybylski et al., 2013), es una escala unidimensional de diez ítems ideada para medir el miedo perderse algo/noticia (en alguna red social), consta de un escalamiento de cinco puntos (1 = para nada cierto, 2 = ligeramente cierto, 3= moderadamente cierto, $4=$ muy cierto, 5= extremadamente cierto). Con fines pertinentes para su uso en este estudio las puntuaciones reportadas fueron $\alpha=.73$ IC 95\% [.70, .74] (M=9.14, DE=3.58).

\section{Procedimiento}

Para continuar coherentemente con los objetivos planteados para el estudio, antes de la aplicación del cuestionario en la muestra objetivo, fue aplicado inicialmente a un grupo de universitarios $(n=10)$ para asegurar la completa comprensión de los ítems. Para la aplicación del cuestionario se propuso coordinar la participación de encuestadores con el departamento académico, donde se informó a las autoridades las implicaciones del estudio y solo se contaría bajo las condiciones de participación voluntaria y anónima en la presente investigación. La escala fue aplicada en grupos entre 30 y 40 estudiantes. Los participantes fueron informados sobre los fines y propósitos del estudio así como del anonimato y duración de la aplicación (aproximadamente 20 minutos). Solo se contó con los participantes que accedieron totalmente de manera voluntaria y no remunerada. El estudio fue aprobado por el Comité de Ética del Instituto Peruano de Orientación Psicológica.

\section{Análisis de datos}

Para los análisis se tomó en cuenta tanto estadísticos descriptivos e inferenciales como la prueba $t$ de Student para evaluar las diferencias entre grupos y la $d$ de Cohen para la Magnitud de Efecto (ME; Cohen, 1988; Kelly y Preacher, 2012). Para evaluar la estructura interna de la escala BFAS fue tomado un enfoque de análisis factorial confirmatorio (AFC). Debido al escalamiento ordinal de los datos para el AFC fueron empleadas matrices policóricas con el método de máxima verosimilitud (Holgado-Tello, Chacón-Moscoso, Barbero-García y Vila-Abad, 2010; Lee, Poon y Bentler, 1998). Para el análisis del modelo de medida fueron utilizados puntos de corte de los índices de bondad de ajuste (Hu y Bentler, 1998) $x^{2}$ corregido por Satorra y Bentler $\left(\mathrm{SB}-x^{2}\right)$ como índice global, para la medida incremental del modelo fue utilizado el índice de ajuste comparativo >.95 (CFI), para la medida de la parsimonia se utilizó la raíz del error cuadrático media de aproximación $\leq .06$ (RMSEA), adicionalmente la raíz promedio de los residuos estandarizados $\leq .08$ (SRMR) y finalmente fue calculada la raíz promedio de los residuos estandarizados ajustada por la parsimonia del modelo 
(PSRMR). Para la estimación de la validez convergente de los ítems se utilizó la varianza promedio extraída (AVE) usando el punto de corte $>.50$ (Fornell y Larcker, 1981). Además se propuso analizar la equivalencia de medición entre los grupos según género, edad y afectación académica aplicando un análisis factorial confirmatorio multigrupo (AFC-MG), en primer lugar para ambas muestras no se aplicaron restricciones (invarianza configural), de manera consecutiva se aplicó restricciones en las cargas factoriales (invarianza métrica), para el siguiente paso se dispuso a restringir de manera sucesiva los interceptos (invarianza fuerte) y finalmente fueron restringidos las varianzas de los errores (invarianza estricta); donde se utilizó como criterio la diferencia sucesiva al aplicar restricciones de CFIs.01 como máximo cambio entre modelos anidados (Cheung y Rensvold, 2002). Para evaluar la fiabilidad de las puntuaciones fueron identificados otros modelos confirmatorios para el uso pertinente del coeficiente alfa (Cronbach, 1951; Graham, 2006), además del cálculo de su versión ordinal debido a la naturaleza de los datos (Zumbo, Gadermann y Zeisser, 2007). Los análisis fueron realizados con el programa EQS 6.2 y SPSS vs.23.

\section{RESULTADOS}

\section{Análisis de ítems}

En la Tabla 1 se muestran las puntuaciones de la media $(M)$, las desviación estándar $(D E)$ y correlación totalcorregida ( $r_{i t c}$ como índice de discriminación de los ítems, además se establecieron valores percentilares (Pc) para la escala BFAS, tomado de referencia el Pc 80 para dividir a los participantes con un uso problemático (Chow, Leung, Ng, y Yu, 2009). La puntuación media más baja fue en el ítem 6 " ¿Sintió el impulso de usar Facebook cada vez más?" (1.77 - 2.01). La mayoría de los ítems evidenciaron valores aceptables de asimetría y curtosis. Asimismo los índices de discriminación obtenidos en la escala BFAS fueron aceptables $\left(r_{\text {itc }}=.534-.632\right)$.

\section{Evidencias de validez basada en la estructura interna}

En la Tabla 2 se evidencia que en todos los casos, los ítems de la escala BFAS presentan pesos factoriales robustos $(\lambda>.60)$, además sus IC 95\% fueron entre $\lambda=.52 \mathrm{y}$ $\lambda=.81$ y la varianza común compartida $\left(\mathrm{h}^{2}\right)$ fue mayor a .50 , resultado contrastado con los valores reportados en otros países ( $\lambda=.59$ a .88), también la convergencia de los ítems fue evaluada por la varianza promedio extraída
(AVE) que fue de .51 (los AVE y la FC para los otros países fueron calculadas). El modelo unidimensional puesto a prueba evidenció un porcentaje adecuado con relación a la retención de varianza capturada por el factor latente, además la fiabilidad compuesta (FC) presenta valores adecuados FC>.80 (Fornell y Larcker, 1981).

Tabla 1. Estadísticos descriptivos para la escala BFAS

\begin{tabular}{|c|c|c|c|c|c|}
\hline BFAS & $M$ & $D E$ & $g_{1}$ & $g_{2}$ & $r_{\text {itc }}$ \\
\hline Ítem 1 & 2.05 & .931 & .728 & .088 & .534 \\
\hline Ítem 2 & 2.02 & .985 & .786 & .051 & .614 \\
\hline Ítem 3 & 2.03 & .990 & .661 & -.353 & .622 \\
\hline Ítem 4 & 2.04 & .988 & .779 & .035 & .591 \\
\hline Ítem 5 & 2.06 & 1.062 & .761 & -.226 & .582 \\
\hline Ítem 6 & 1.90 & .938 & .874 & .146 & .616 \\
\hline \multicolumn{6}{|l|}{$\mathrm{Pc}$} \\
\hline$<80$ & 384 & $75.3 \%$ & & & \\
\hline $\begin{array}{l}80 \quad a \\
\text { más }\end{array}$ & 126 & $24.7 \%$ & & & \\
\hline
\end{tabular}

Tabla 2. Pesos factoriales a nivel internacional

\begin{tabular}{lccccccc}
\hline Ítems & $\mathrm{P} \lambda$ & $\lambda \mathrm{IC} 95 \%$ & $h^{2}$ & $\mathrm{~N} \lambda$ & $\mathrm{PO} \lambda$ & $\mathrm{T} \lambda$ & $\mathrm{P} . \mathrm{A} . \lambda$ \\
\hline 1 & 0.62 & $(0.54 ; 0.69)$ & 0.519 & 0.61 & 0.54 & 0.83 & 0.58 \\
2 & 0.74 & $(0.64 ; 0.79)$ & 0.715 & 0.80 & 0.67 & 0.86 & 0.64 \\
3 & 0.75 & $(0.69 ; 0.81)$ & 0.683 & 0.59 & 0.72 & 0.78 & 0.43 \\
4 & 0.71 & $(0.62 ; 0.78)$ & 0.603 & 0.67 & 0.54 & 0.79 & 0.64 \\
5 & 0.70 & $(0.64 ; 0.76)$ & 0.593 & 0.69 & 0.69 & 0.86 & 0.66 \\
6 & 0.74 & $(0.67 ; 0.79)$ & 0.595 & 0.69 & 0.77 & 0.88 & 0.59 \\
& & & & & & & \\
FC & 0.86 & & & 0.83 & 0.82 & 0.93 & 0.76 \\
AVE & 0.51 & & & 0.46 & 0.43 & 0.68 & 0.35 \\
\hline
\end{tabular}

Nota: $\lambda=$ cargas factoriales; $\mathrm{P}=$ Perú; $\mathrm{N}=$ Noruega; $\mathrm{PO}=$ Portugal; $\mathrm{T}=$ Tailandia; P.A.=Países Árabes; AVE= varianza promedio extraída; $h^{2}=$ comunalidad; FC= Fiabilidad compuesta

Por ello, con base a lo identificado en estudios previos se tomó en cuenta la unidimensionalidad del modelo (Ahmet et al., 2017; Andreassen, 2015; Andressen et al., 2012, Phanasathit et al., 2015; Pontes et al., 2016) donde se verificó indicadores satisfactorios en la mayoría de índices de bondad de ajuste SB-x2=23.9315(9), CFI=.994, TLI=.990, SRMR=.032, RMSEA=.061 (IC90\%=.032, .092) corroborando un ajuste unidimensional. Se tomó en cuenta los índices de bondad de ajuste reportados por 
Noruega, Portugal y Turquía (Ahmet et al., 2017; Andreassen et al., 2012; Pontes et al., 2016) para las diferencias en los CFI y RMSEA ( $\triangle \mathrm{CFI}$; $\triangle \mathrm{RMSEA}$ ), que no mostraron diferencias sustanciales respecto al modelo propuesto (ver Tabla 3).

Tabla 3. Diferencias de los índices de bondad de ajuste según país

\begin{tabular}{lcccc}
\hline Países & CFI & RMSEA & $\Delta$ CFI & $\Delta$ RMSEA \\
\hline Perú & 0.994 & 0.061 & - & - \\
Noruega & 0.990 & 0.050 & 0.004 & 0.01 \\
Portugal & 0.992 & 0.032 & 0.002 & 0.003 \\
Turquía & 0.980 & 0.064 & 0.010 & 0.003 \\
\hline
\end{tabular}

Nota: diferencia de $\mathrm{CFI}=\Delta \mathrm{CFI}$; diferencia de RMSEA $=\triangle \mathrm{RMSEA}$

\section{Equivalencia de medición según variables demográficas}

Se analizó la equivalencia de medición (o invarianza factorial) entre el grupo de varones $\left(S_{1}\right)$ y mujeres $\left(S_{2}\right)$, el modelo base puesto a prueba para la invarianza configural $\left(S_{1} \wedge=S_{2} \wedge\right.$ ) evidenció índices de bondad de ajuste satisfactorios, $C F I=.998, R M S E A=.015$ (IC 90\%= .000; .071). Siendo coherente la dimensionalidad en ambos grupos se pasó a la siguiente etapa que fue analizar la invarianza métrica, CFI=.999, RMSEA=.00 (IC 90\%= .00; .051) con índices también satisfactorios. Estos resultados mostraron que ambos grupos son invariantes respecto a la métrica de sus ítems, donde la diferencia establecida es óptima $\left(\Delta_{C F I}=.001\right)$ (Cheung y Rensvold, 2002). En el siguiente nivel se evaluó la invarianza fuerte, $C F I=.996$, RMSEA=.021 (IC 90\%=.000; .060), donde la diferencia de ambos modelos fue $\Delta_{C F I}=.003$, apreciándose por consiguiente el cumplimiento del modelo a pesar de las restricciones. Debido a que se mantuvo este nivel de invarianza, se pasó a evaluar la invarianza estricta, CFI=.996, RMSEA=.018 (IC 90\%=.00; .055), esta diferencia entre ambos modelos fue $\Delta_{C F}=.001$, por lo que se mantienen invariantes los residuales entre ambos grupos.
También se dispuso evaluar la equivalencia de medición entre el grupo menores de 20 años $\left(E_{1}\right)$ y mayores de 20 años $\left(E_{2}\right)$, la invarianza configural $\left(E_{1} \wedge=E_{2}\right.$ $\Lambda_{\mathrm{g}}$ ) evidencio índices de bondad de ajuste satisfactorios, $C F I=.992$, RMSEA=.065 (IC 90\%=.033; .096). Por ello, se pasó a la siguiente etapa para analizar la invarianza métrica, con resultados aceptables aunque hayan sido impuestas las restricciones CFI=.986, RMSEA=.072 (IC $90 \%=.046 ; .098)$. Estos resultados mostraron que ambos grupos son invariantes respecto a la métrica de sus ítems, donde la diferencia establecida es óptima $\left(\Delta_{C F I}=.006\right)$. Para la evaluación de la invarianza fuerte se encontró que al imponer nuevas restricciones el modelo se deteriora significativamente CFI=.95, RMSEA=.076 (IC 90\%= .056; .106), la diferencia de ambos modelos fue $\Delta_{C F l}=.03$, donde no se pudo corroborar este nivel de invarianza.

El último grupo evaluado para la equivalencia de medición fuel el que ha sido afectado en su rendimiento académico $\left(A_{1}\right)$ y el no afectado $\left(A_{2}\right)$, nuevamente la invarianza configural brinda un buen ajuste $C F I=.991$, $R M S E A=.062$ (IC 90\%= .027; .094) y la invarianza métrica obtuvo índices aceptables CFI=.99, RMSEA=.055 (IC 90\%= .023; .086), donde la diferencia entre grupos fue ( $\Delta_{C F I}$ $=.001$ ). Sin embargo la invarianza estricta también evidenció un deterioro del modelo en este grupo $C F I=.96$, RMSEA $=.070$ (IC 90\%=.042; .096, al ser impuestas restricciones en los interceptos, donde el modelo no superó el punto de corte establecido con una diferencia entre grupo de $\Delta C F=.027$ por lo que no se optó por pasar al siguiente nivel de invarianza.

\section{Evidencias basadas en relación con otras variables}

La correlación entre las puntuaciones derivadas de la escala BFAS y el FoMO fue positiva $(r=.60)$ con una ME grande $\left(r^{2}=.36\right)$ para la puntuación general. La diferencia en las puntuaciones de los usuarios con uso problemático y no problemático asociados a Facebook fue estadísticamente significativa ( $\mathrm{t}=13.34 ; p<.001)$ y la magnitud de la diferencias fue grande (ver Tabla 4).

Tabla 4. Relación entre conductas problemática de FoMo y puntuaciones del BFAS

\begin{tabular}{|c|c|c|c|c|c|c|c|}
\hline & $\begin{array}{l}\text { Conducta problemática } \\
\text { (FoMO) }\end{array}$ & $n$ & ME (DS) & $t$ & $p$ & $d$ & IC 95\% \\
\hline BFAS & No & 388 & $10.15(2.59)$ & 13.34 & $<.001$ & 1.38 & {$[1.16 ; 1.61]$} \\
\hline
\end{tabular}

Nota: $d=\mathrm{d}$ de Cohen como medida de ME 


\section{Estudios de diferencias según género y afectación social en universitarios}

La diferencia de las puntuaciones observadas entre grupos fue analizada con la prueba t de student (t), para este análisis se evaluaron los grupos según género, afectación en el rendimiento académico, afectación a nivel de relaciones interpersonales y afectación a nivel familiar. El grupo según género evidenció una diferencia estadísticamente significativa $(t=-2.09 ; p=.036)$ pero a nivel de significancia práctica, la magnitud del efecto (ME) fue trivial $d=.18 \mathrm{IC} 95 \%[.01, .36]$. En el grupo de afectación a nivel académico las diferencias fueron estadísticamente significativas $(t=-6.69 ; p<.001)$ la ME fue grande $d=.74$ con IC 95\% [.50, .98]. Para el grupo de afectación a nivel relaciones interpersonales las diferencias fueron estadísticamente significativas $(t=-5.566 ; p<.001)$ la $\mathrm{ME}$ fue mediana $d=.58$ con IC $95 \%[.38, .80]$ y finalmente el grupo de afectación a nivel de relaciones familiares fue también estadísticamente significativa $(t=-6.699 ; p<.001)$ la ME fue mediana $d=.65$ con IC 95\% $[.47, .86]$

\section{Fiabilidad de las puntuaciones del BFAS}

En la Tabla 5 se presenta el análisis de la fiabilidad del modelo para el BFAS. Los análisis confirman que la diferencia entre el modelo tau-equivalente (M2) y el congenérico (M1), es mínima incluso si se comparase con el modelo paralelo (M3), esto indicaría que tanto conceptos (M1) como medias y covarianzas (M2) son similares. Por ello el modelo tau-equivalente puede representar satisfactoriamente el modelo de fiabilidad. Basado en estos resultado fue empleado el coeficiente de consistencia interna alfa de Cronbach (a; Cronbach, 1951; Graham, 2006) y sus intervalos de confianza [IC 95\%] con el método de Feldt para el cálculo de la fiabilidad de las puntuaciones (Feldt y Brennan, 1989; Romano, Kromrey, Owens, y Scott, 2011), que presentaron óptimos resultados tomando en cuenta la naturaleza ordinal de los datos $a_{\text {ordinal }}=.847$ [IC95\% .818, .871] (Zumbo et al., 2007), la correlación inter ítem promedio fue $\left(r_{\mathrm{ij}}\right) .47$ y el error estándar de medida (EEM) 1.68.

Tabla 5. Estructura interna de la escala BFAS para el modelo de fiabilidad

\begin{tabular}{|c|c|c|c|c|c|c|c|}
\hline & SB $x^{2}(g l)$ & $\mathrm{CFI}$ & TLI & SRMR & PSRMR & RMSEA & (IC 90\%) \\
\hline M1 & 23.9315 (9) & .994 & .99 & .032 & .048 & .061 & {$[.032, .092]$} \\
\hline \multicolumn{8}{|c|}{ Modelo de fiabilidad } \\
\hline \multirow[t]{2}{*}{ M2 } & $29.6566(14)$ & .993 & .993 & .040 & .048 & .050 & {$[.025 ; .076]$} \\
\hline & & $\Delta \mathrm{CFI}(.001)$ & $\Delta \mathrm{TLI}(.003)$ & & & $\triangle$ RMSEA (.01) & \\
\hline \multirow[t]{2}{*}{ M3 } & $40.85(19)$ & .965 & .963 & .064 & .067 & .051 & {$[.029 ; .073]$} \\
\hline & & $\Delta$ CFI (.02) & $\Delta \mathrm{TLI}(.02)$ & $\Delta_{\text {SMRS }}(.03)$ & & $\triangle$ RMSEA $(.01)$ & \\
\hline
\end{tabular}

Nota: M1 = modelo congenérico; M2= modelo tau-equivalente; M3= Modelo paralelo; CFI/TLI=índices de ajuste comparativo; TLI=; SRMR= raíz promedio de los residuos estandarizados PSRMR= raíz del residuo estandarizado cuadrático media ajustada por la parsimonia; RMSEA=raíz cuadrática del error de aproximación; $\triangle \mathrm{CFI}=$ diferencia entre $\mathrm{CFI} ; \mathrm{RMSEA}=$ diferencia entre RMSEA

\section{DISCUSIÓN}

Debido al creciente aumento de las adicciones comportamentales, en especial las adicciones tecnológicas como la adicción a Facebook, es importante que los profesionales de la salud mental cuenten con un instrumento adecuado y preciso que contribuya con el diagnóstico temprano para esta adicción. A pesar de ser una escala de uso habitual en relación a la adición a Facebook en el continente europeo, el presente estudio ofrece la primera versión en idioma español de la escala. Los objetivos planteados para este estudio fueron analizar la estructura interna del instrumento, la fiabilidad de las puntuaciones y la equivalencia de las medidas en el contexto peruano.
Los resultados del análisis factorial confirmatorio apoyan la unidimensionalidad de la escala para este estudio, que replicó la solución de un solo factor con cargas factoriales robustas similares a los estudios reportados en Noruega, Portugal y Tailandia (Andreassen, 2015; Andreassen et al., 2012, Phanasathit et al., 2015; Pontes et al., 2016). La varianza capturada por el factor latente representó el 51\% que indica el porcentaje de varianza sustancial explicada por el constructo. Comparado con estudios internacionales, solo el estudio de Tailandia logró un 68\% siendo el que obtuvo mejor representatividad (Phanasathit et al., 2015) 
seguido por el estudio peruano. Estos resultados pueden sustentar la estabilidad de la estructura interna del BFAS.

En este estudio se encontró una fuerte relación entre el BFAS y el FoMO, es decir que el miedo a perderse alguna noticia en Facebook o cualquier red social podría ser un predictor de esta adicción (Alt, 2015; Dias et al., 2018; Przybylski et al., 2013), debido a las características potencializadoras de la conducta de conexión que mantiene el comportamiento habitual de participación en las RSV (Abel et al., 2012). Esto representaría el 36\% de la influencia de la varianza de una variable en atribución a la otra (Cohen, 1988; Kelly y Preacher, 2012). Además las diferencias entre usuarios con alto FoMO (conducta problemática) con las puntuaciones totales del BFAS fueron estadísticamente significativas donde puede atribuirse esta variable como un potenciador del uso problemático de Facebook (Chow et al., 2009).

La evaluación de la equivalencia de medición entre diferentes grupos como la edad, género o que presenten alguna repercusión a nivel personal, social o familiar es importante debido a que al analizar factores de riesgo del comportamiento adictivo, los análisis de invarianza factorial comparan tanto ítems como variables latentes que puedan medir el mismo significado para estos grupos. La equivalencia de medición fue evaluada mediante AFC-MG según género, donde se cumplió los supuestos de invarianza configural, métrica, fuerte y estricta (Cheung y Resvold, 2002; Vandenberg y Lance, 2000; Widaman y Reise, 1997), ello implicaría que los ítems de BFAS como la estructura latente tienen el mismo significado para este grupo en particular. Sin embargo la evaluación de los grupos de edad y la afectación a nivel académico solo se cumplieron los supuestos de invarianza configural y métrica (configuración de ítems y cargas factoriales). Esto implicaría que para la edad y afectación académica las diferencias promedio en la variable latente no capturarían todas las diferencias promedio en la varianza compartida siendo estas parcialmente aceptables (Putnick y Bornstein, 2016). Este hallazgo es un indicador y de utilidad para contextos clínicos (como las conductas adictivas) debido a que lo ideal es llegar al último nivel de invarianza para una adecuada discriminación del constructo en diferentes grupos que pueda tener en cuenta tratamientos diferenciados, como es el caso de las adicciones convencionales y con menor medida otras adicciones comportamentales actualmente más estudiadas como la adicción al trabajo y videojuegos (Beiler-May et al., 2016), pero en este estudio las diferencias fueron parciales con relación a la edad y la afectación a nivel académico.

Al analizar la diferencia de las variables observables, la variable sexo fue establecida debido a las diferencias específicas en cuanto el comportamiento adictivo (Becker et al., 2017), donde sí evidencia una mínima diferencia pero la magnitud de estas diferencias fue trivial, esto no implicaría una magnitud de diferencias real entre ambos grupos. En cuanto a las diferencias entre las variable con mayor distanciamiento, el sentirse afectado nivel académico y los problemas a nivel de relaciones interpersonales (social y familiar) por el uso prolongado de Facebook presentaron discrepancias marcadas entre las puntuaciones de los grupos. Esto implicaría un factor de riesgo en el uso prolongado a Facebook en estos grupos que podría desencadenar y/o mantener trastornos psicopatológicos como conductas problemáticas a nivel de concentración, búsqueda de sensaciones, ansiedad social y sintomatología depresiva (Hormes et al., 2014; Meier et al., 2016; Moreau et al., 2015).

La fiabilidad de las puntuaciones de la escala BFAS fue adecuada bajo la evaluación de los supuestos sobre su uso pertinente del coeficiente alfa, los modelos tauequivalentes y medidas paralelas fueron puestos a prueba donde una alta uniformidad de cargas que representa el factor latente además de un deterioro no significativo a nivel estructural, incluso los errores de medida aleatorios son similares, superando el estándar (a>.70). En adición, el promedio de las correlaciones interítem $\left(r_{\mathrm{ij}}\right)$ se relacionan de idéntica manera y grado a la varianza total, estos resultados corroboran el uso adecuado de la BFAS para la investigación. A pesar de ello, en este estudio no pudo evidenciarse un rango de precisión para contextos de evaluación clínica donde es requerido un a>90 (Bland y Altman, 1997; Mayo, 2015).

A pesar que los resultados de este estudio proporcionan apoyo a nivel psicométrico para la versión en español del BFAS, deben ser reconocidas algunas limitaciones como la medida de equivalencia entre otros contextos hispanohablantes (estudios interculturales), mayores rangos de edad y condición laboral, así como la evaluación de puntos de corte. Dentro de esta línea, los autores están encaminados hacia futuros estudios en la evaluación la versión en español del BFAS con muestras de mayor diversidad (por ejemplo parejas, trabajadores, escolares y nivel cultural), que puedan ser útiles para la investigación en las adicciones comportamentales. 
En conclusión, es necesario contar con evidencias de validez y fiabilidad en idioma español del BFAS que resulten útiles para la investigación y diagnóstico temprano de la adicción a Facebook, además de equipar de una herramienta útil y rápida a los profesionales de la salud. En particular esta escala puede ser incluida en los protocolos de evaluación y servir como un instrumento de apoyo a otras técnicas de evaluación que contribuya con evidencia empírica para el diagnóstico del uso problemático y adictivo del Facebook.

\section{Reconocimientos}

Los autores expresan su agradecimiento a la Dra. Domitila Guerrero-Vigil por su dedicación al estudio de las relaciones familiares y al Mg. César Merino-Soto por sus valiosas recomendaciones en el proceso instrumental.

\section{Conflicto de interés}

Los autores de este artículo declaran no tener ningún conflicto de interés

\section{REFERENCIAS}

Abel, J. P., Buff, C. L., y Burr, S. A. (2016). Social media and the fear of missing out: Scale development and assessment. Journal of Business \& Economics Research, 14(1), 33-43.

Ahmet, A. K. I. N., Demirci, I., y Serap, K. A. R. A. (2017). Facebook bağimliliği ölçeği nin türkçe formunun geçerliği ve güvenirliği. Akademik Bakış Uluslararası Hakemli Sosyal Bilimler Dergisi, (59), 65-72

Alt, D. (2015). College students' academic motivation, media engagement and fear of missing out. Computers in Human Behavior, 49, 111-119. doi: 10.1016/j.chb.2015.02.057

Andreassen, C. S. (2015). Online social network site addiction: A comprehensive review. Current Addiction Reports, 2(2), 175-184. doi: 10.1007/s40429-015-0056-9

Andreassen, C. S., Torsheim, T., Brunborg, G. S., y Pallesen, S. (2012). Development of a Facebook addiction scale. Psychological Reports, 110(2), 501-517. doi: 10.2466/02.09.18.PR0.110.2.501-517

Becker, J. B., McClellan, M. L., y Reed, B. G. (2017). Sex differences, gender and addiction. Journal of neuroscience research, 95(1-2), 136-147. doi: 10.1002/jnr.23963

Becoña, E., y Cortés, M. (2016). Manual de adicciones para psicólogos especialistas en psicología clínica en formación. Barcelona: Sociodrogalcohol

Beiler-May, A., Williamson, R. L., Clark, M. A., y Carter, N. T. (2017). Gender bias in the measurement of workaholism. Journal of Personality Assessment, 99(1), 104-110. doi: 10.1080/00223891.2016.1198795
Bland, J. M., y Altman, D. G. (1997). Cronbach's alpha. BMJ: British Medical Journal, 314(7080), 572. doi: 10.1136/bmj.314.7080.572

Brailovskaia, J., y Margraf, J. (2017). Facebook Addiction Disorder (FAD) among German students - A longitudinal approach. Plos One, 12(12) 1-15. doi: 10.1371/journal.pone.0189719.g001

Casale, S., y Fioravanti, G. (2018). Why narcissists are at risk for developing Facebook addiction: The need to be admired and the need to belong. Addictive behaviors, 76, 312-318. doi: 10.1016/j.addbeh.2017.08.038

Chan, T. K., Cheung, C. M., Shi, N., y Lee, M. K. (2015). Gender differences in satisfaction with Facebook users. Industrial Management \& Data Systems, 115(1), 182-206. doi: 10.1108/IMDS-08-2014-0234

Cheung, G. W., y Rensvold, R. B. (2002). Evaluating goodness-of-fit indexes for testing measurement invariance. Structural equation modeling, 9(2), 233-255. doi: 10.1207/S15328007SEM0902_5

Chow, S. L., Leung, G. M., Ng, C., y Yu, E. (2009). A screen for identifying maladaptive internet use. International Journal of Mental Health and Addiction, 7(2), 324-332.

Cohen, J. (1988). Statistical power analysis for the behavioral sciences (2nd ed.). New York, NY: Academic Press.

Cronbach, L. J. (1951). Coefficient alpha and the internal structure of tests. psychometrika, 16(3), 297-334.

Dhaha, I. S. Y. (2013). Predictors of Facebook addiction among youth: A Structural Equation Modeling (Sem). Journal of Social Sciences (COES\&RJ-JSS), 2(4), 186-195.

Dias, P., Cadime, I., del Castillo-López, A., García-Castillo, F., y García de Castillo, J. (2018). Excessive facebook use among portuguese university students: contributes of the adaptation of the Bergen facebook addiction. Salud y Drogas, 18(1), 131-139. doi: 10.21134/haaj.v18i1.377

Drouin, M., O'Connor, K. W., Schmidt, G. B., y Miller, D. A. (2015). Facebook fired: Legal perspectives and young adults' opinions on the use of social media in hiring and firing decisions. Computers in Human Behavior, 46, 123-128. doi: 10.1016/j.chb.2015.01.011

El Sahili, L. (2015). Psicología de facebook: Vislumbrando los fenómenos psíquicos, complejidad y alcance de la red social más grande del mundo. México: Trillas.

Feldt, L. S., y Brennan, R. L. (1989). Reliability. In R. L. Linn (Ed.), Educational measurement (3rd ed., pp. 105-146). New York, NY: American Council on Education and Macmillan.

Fornell, C., y Larcker, D. F. (1981). Evaluang structural equation models with unobservable variables and measurement error. Journal of Markeng Research, 18, 39- 50. doi: 10.2307/3151312

Garcia del Castillo, J. (2013). Technological addictions: the rise of socia networks. Salud y Drogas, 13(1), 5-14. doi: 10.21134/haaj.v13i1.188

Graham, J. M. (2006). Congeneric and (essentially) tau-equivalent estimates of score reliability: What they are and how to use them. Educational and psychological measurement, 66(6), 930-944. doi: $10.1177 / 0013164406288165$ 
Holgado-Tello, F. P., Chacón-Moscoso, S., Barbero-García, I., y Vila-Abad, E. (2010). Polychoric versus Pearson correlaons in exploratory and confirmatory factor analysis of ordinal variables. Quality \& Quanty, 44(1), 153-166. doi: 10.1007/s11135-008-9190-y

Hormes, J. M., Kearns, B., y Timko, C. A. (2014). Craving Facebook? Behavioral addiction to online social networking and its association with emotion regulation deficits. Addiction, 109(12), 2079-2088. doi: 10.1111/add.12713

Hu, L. T., y Bentler, P. M. (1998). Fit indices in covariance structure modeling: Sensitivity to underparameterized model misspecification. Psychological methods, 3(4), 424. doi: 10.1037/1082-989x.3.4.424

Kelley, K., y Preacher, K. J. (2012). On effect size. Psychological methods, 17(2), 137-152. doi:10.1037/a0028086

Lee, S.-Y., Poon, W.-Y., y Bentler, P. M. (1995). A two-stage esmaon of structural equaon models with connuous and polytomous variables. Brish Journal of Mathemical and Stascal Psychology, 48(2), 339-358. doi: 10.1007/BF02294660

Mayo, A. M. (2015). Psychometric instrumentation: reliability and validity of instruments used for clinical practice, evidence-based practice projects and research studies. Clinical nurse specialist, 29(3), 134 138. doi:10.1097/NUR.0000000000000131

Meier, A., Reinecke, L., y Meltzer, C. E. (2016). "Facebocrastination"? Predictors of using Facebook for procrastination and its effects on students' well-being. Computers in Human Behavior, 64, 65-76. doi: 10.1016/j.chb.2016.06.011

Moreau, A., Laconi, S., Delfour, M., y Chabrol, H. (2015). Psychopathological profiles of adolescent and young adult problematic Facebook users. Computers in Human Behavior, 44, 64-69. doi: 10.1016/j.chb.2014.11.045

Phanasathit, M., Manwong, M., Hanprathet, N., Khumsri, J., y Yingyeun, R. (2015). Validation of the Thai version of Bergen Facebook addiction scale (Thai-BFAS). Journal of the Medical Association of Thailand, 98(2), S108-S117.

Muñiz, J., Elosua, P., y Hambleton, R. K. (2013). International Test Commission Guidelines for test translation and adaptation. Psicothema, 25(2), 151-157. doi: 10.7334/psicothema2013.24

Ortuño-Sierra, J., Aritio-Solana, R., y Fonseca-Pedrero, E. (2018). Mental health difficulties in children and adolescents: The study of the SDQ in the Spanish National Health Survey 2011-2012. Psychiatry research, 259, 236-242. doi: 10.1016/j.psychres.2017.10.025

Pontes, H. M., Andreassen, C. S., y Griffiths, M.D. (2016). Portuguese Validation of the Bergen Facebook Addiction Scale: an Empirical Study. International Journal of Mental Health Addiction, 14, 10621073. doi: 10.1007/s11469-016-9694-y

Przybylski, A. K., Murayama, K., DeHaan, C. R., y Gladwell, V. (2013). Motivational, emotional, and behavioral correlates of fear of missing out. Computers in Human Behavior, 29(4), 1841-1848. doi: 10.1016/j.chb.2013.02.014
Putnick, D. L., y Bornstein, M. H. (2016). Measurement invariance conventions and reporting: the state of the art and future directions for psychological research. Developmental Review, 41, 71-90.

Roberts, J. A., y David, M. E. (2016). My life has become a major distraction from my cell phone: Partner phubbing and relationship satisfaction among romantic partners. Computers in Human Behavior, 54, 134 141. doi: $10.1016 / j . c h b .2015 .07 .058$

Romano, J. L., Kromrey, J. D., Owens, C. M., y Scott, H. M. (2011). Confidence interval methods for coefficient alpha on the basis of discrete, ordinal response items: Which one, if any, is the best? The Journal of Experimental Education, 79 (4) , 382 - 403. doi: 10.1080/00220973.2010.510859

Satici, S. A., y Uysal, R. (2015). Well-being and problematic Facebook use. Computers in Human Behavior, 49, 185-190. doi: 10.1016/j.chb.2015.03.005

Salem, A.A., Almenaye, N.S., y Andreassen, C.S. (2016). A Psychometric Evaluation of Bergen Facebook Addiction Scale (BFAS) of University Students. International Journal of Psychology and Behavioral Sciences, 6(5), 199-205. doi: 10.5923/j.ijpbs.20160605.01

Silva, H., Areco, K.C., Bandiera-Paiva, P., Galvão, P.V., Garcia, A.N., y Silveira, D.X. (2015). Equivalência semântica e confiabilidade da versão em português da Bergen Facebook Addiction Scale. Jornal Brasileiro de Psiquiatria, 64(1), 17-23. doi: 10.1590/0047-2085000000052

Vandenberg, R. J., y Lance, C. E. (2000). A review and synthesis of the measurement invariance literature: Suggestions, practices, and recommendations for organizational research. Organizational research methods, 3(1), 4-70. doi: 10.1177/109442810031002

Wang, C. W., Ho, R. T., Chan, C. L., y Tse, S. (2015). Exploring personality characteristics of Chinese adolescents with internet-related addictive behaviors: Trait differences for gaming addiction and social networking addiction. Addictive behaviors, 42, 32-35. doi: 10.1016/j.addbeh.2014.10.039

Widaman, K. F., y Reise, S. P. (1997). Exploring the measurement invariance of psychological instruments: Applications in the substance use domain. In K. J. Bryant, M. Windle, y S. G. West (Eds.), The science of prevention: Methodological advances from alcohol and substance abuse research (pp. 281-324). Washington, DC, US: American Psychological Association. doi: 10.1037/10222-009

Yildirim, C., y Correia, A. P. (2015). Exploring the dimensions of nomophobia: Development and validation of a self-reported questionnaire. Computers in Human Behavior, 49, 130-137. doi: 10.1016/j.chb.2013.02.014

Young, N. L., Kuss, D. J., Griffiths, M. D., y Howard, C. J. (2017). Passive Facebook use, Facebook addiction, and associations with escapism: An experimental vignette study. Computers in Human Behavior, 71 , 24-31. doi: 10.1016/j.chb.2017.01.039

Zumbo, B. D., Gadermann, A. M., y Zeisser, C. (2007). Ordinal versions of coefficients alpha and theta for Likert rating scales. Journal of Modern Applied Statistical Methods, 6(1), 4. doi: 10.22237/jmasm/1177992180 